\title{
Reflets
}

Revue ontaroise d'intervention sociale et communautaire

\section{L'intégration sociale : la lumière au bout du tunnel — Entrevue avec Joanne Beauchamps, Margaret Borley et Louise Grégoire}

\section{Stéphane Laberge}

Volume 4, numéro 2, automne 1998

Personnes vivant avec une incapacité

URI : https://id.erudit.org/iderudit/026215ar

DOI : https://doi.org/10.7202/026215ar

Aller au sommaire du numéro

Éditeur(s)

Reflets : Revue ontaroise d'intervention sociale et communautaire

ISSN

1203-4576 (imprimé)

1712-8498 (numérique)

Découvrir la revue

Citer ce document

Laberge, S. (1998). L'intégration sociale : la lumière au bout du tunnel Entrevue avec Joanne Beauchamps, Margaret Borley et Louise Grégoire. Reflets, 4(2), 20-30. https://doi.org/10.7202/026215ar

Tous droits réservés (C) Reflets : Revue ontaroise d'intervention sociale et communautaire, 1998
Ce document est protégé par la loi sur le droit d'auteur. L'utilisation des services d'Érudit (y compris la reproduction) est assujettie à sa politique d'utilisation que vous pouvez consulter en ligne.

https://apropos.erudit.org/fr/usagers/politique-dutilisation/ 


\section{L'intégration sociale : la lumière au bout du tunnel}

\section{Entrevue avec Joanne Beauchamps, Margaret Borley et Louise Grégoire}

L'entrevue présentée dans ce numéro de Reflets porte sur l'intégration sociale des personnes vivant avec une incapacité. Elle résume, par les témoignages de Joanne Beauchamps et de Louise Grégoire, les difficultés et les défis à surmonter pour quiconque vit quotidiennement avec une incapacité. Elle fait aussi état de certains mythes et préjugés que la société porte à l'égard de ces personnes. De plus, les propos de Margaret Borley montrent comment il est possible, malgré les embûches, de faciliter l'intégration sociale des personnes vivant avec une incapacité.

Le résumé de ces entrevues a été effectué par Stéphane Laberge, reporter à la télévision de Radio-Canada

\section{L'intégration sociale : les personnes ayant une inca- pacité voient poindre la lumière au bout du tunnel}

Je veux me marier et construire une maison sur un terrain que nous avons acheté mon petit ami et moi.Je tiens aussi à poursuivre mes études en informatique.

Ce sont les rêves de Louise Grégoire de Sudbury. Elle fera tout pour les réaliser même si elle est atteinte de sclérose en plaques, ce qui l'oblige à se déplacer en fauteuil roulant. Sa détermination, son courage, Louise Grégoire les tient d'un défi très difficile qu'elle a su surmonter, celui d'accepter de vivre avec une incapacité et 
de prendre sa place au sein de la communauté. Elle dira :

J'avais un emploi à la station de radio CHNO où $j$ 'ai travaillé pendant cinq ans. Malheureusement, $j$ 'ai dû quitter mon poste car j'étais trop malade, j'étais atteinte de la sclérose en plaques et je croyais que ma vie était désormais finie!

À l'âge de 24 ans, bien que cela n'ait pas toujours été facile, car les embûches ont été nombreuses, elle a commencé sa bataille.

Le cas de Joanne Beauchamps est un peu différent. Originaire de Timmins et atteinte de cécité visuelle, ses parents préparaient tout pour elle et la protégeait constamment. Joanne ne faisait jamais rien toute seule. Les gens la prenaient en pitié, car dès son jeune âge, elle devait marcher avec une canne.

Je restais enfermée à la maison à ne rien faire. J'étais un peu comme un objet, un bibelot qu'on plaçait dans un endroit de la maison, car j'étais différente des autres personnes.

Louise Grégoire considère avoir tout perdu à cause de la maladie. Son mari l'a laissé parce que la charge de travail et les responsabilités lui étaient trop lourdes à supporter. Et c'est cette image qu'elle verra dans le miroir.

Je refusais de voir une personne confinée à un fauteuil roulant, car je ne voulais pas voir la réalité en face. Quand je regardais quelqu'un dépérir dans un fauteuil roulant, c'est moi que je voyais! C'était la peur de voir quelqu'un vivant avec une incapacité physique.

\section{Les projets de participation communautaire du district de Sudbury: la transition vers un monde meilleur}

Les vies de Louise Grégoire et de Joanne Beauchamps ont complètement changé lorsqu'elles ont décidé de se prendre en 
main, de laisser tomber toutes les barrières psychologiques pour demander de l'aide aux intervenants et aux intervenantes du Centre de participation communautaire de Sudbury. Ce centre a ouvert ses portes en 1982 et est financé partiellement par le ministère de la Santé, dans le cadre des programmes de soins de longue durée. Il vient en aide aux gens atteints d'un handicap physique qui les oblige, dans la plupart des cas, à vivre dans un fauteuil roulant.

En 1979, nous avons créé un conseil d'administration constitué de gens de la communauté de Sudbury. Plusieurs de ces membres avaient dans leur famille une personne qui vivait avec une incapacité physique. Ils voulaient trouver une alternative aux fameuses institutions éloignées où les gens souffrant d'un handicap devaient aller vivre en permanence. Personne ne croyait possible de pouvoir les aider à vivre normalement dans la société.

La directrice du Centre de projets de participation communautaire de Sudbury, Margaret Borley, garde de biens mauvais souvenirs de cette époque où les familles et les médecins envoyaient les personnes atteintes d'un handicap physique ou psychologique dans une résidence destinée uniquement aux gens vivant avec une incapacité.

Les médecins disaient aux parents qu'ils ne pouvaient pas prendre soin de leur enfant atteint d'un handicap et que celui-ci serait mieux dans une institution, finalement un peu comparable à une prison. On les isolait dans des chambres et dans un endroit à l'abri des regards de la société. Pendant plusieurs années, les gens vivant avec une incapacité étaient ni plus ni moins punis et mis de côté parce que la société les hébergeaient dans des institutions bien souvent éloignées de leur famille et de leur communauté.

Le Centre pour la participation communautaire de Sudbury est unique en Ontario. En effet, dans la province, s'il existe d'autres établissements semblables ayant pour rôle de faciliter la réintégration communautaire des personnes souffrant d'une incapacité, celui de Sudbury est le seul à offrir une gamme de programmes comme les logements d'appoint, un centre de jour pour 
l'évaluation et la formation des personnes vivant avec une incapacité, des services d'approche et de relève externe. Le Centre dessert toute la population du Nord ontarien. Actuellement, les seules limites à son mandat sont d'ordre géographique. En effet, les distances séparant certaines villes et la ville de Sudbury où le Centre a son port d'attache, sont tellement grandes que cela complique le travail des conseillers et pose des difficultés quant au service d'approche, comme le souligne Margaret Borley:

Les gens qui utilisent nos services doivent parfois parcourir des distances énormes et dans bien des cas, c'est tout simplement impossible. Voilà pourquoi il faut davantage d'énergie afin d'établir de meilleurs programmes et pour tenter de rejoindre le plus grand nombre de personnes dans leurs milieux.

Au cours des dernières années, les différents paliers de gouvernement ont investi beaucoup d'argent pour instaurer des centres de logement d'appoint pour les personnes ayant une incapacité. Le virage ambulatoire entrepris au cours des dernières années, a forcé le ministère de la Santé à réintégrer les gens au sein de la société. Cette ré-allocation des ressources, à l'ère des compressions budgétaires, a signifié la fermeture des fameuses institutions où l'on enfermait les personnes souffrant d'incapacité physique ou mentale.Aux dires de Margaret Borley, ce fut une excellente chose.

La réduction des budgets aura été bénéfique pour les personnes vivant avec une incapacité. La société s'est finalement aperçue qu'elle pouvait désormais faire quelque chose pour ceux et celles qui cherchaient à prendre leur place parmi elle. La tâche est cependant difficile, car ces gens ont été enfermés pendant de si nombreuses années qu'ils doivent pratiquement tout apprendre ou réapprendre. En effet, durant leur séjour en institution, ce sont les infirmières et les infirmiers qui ont toujours scruté leurs moindres pensées et leurs moindres gestes.

Peu à peu, on a mis en place des programmes de réintégration sociale qui ont fourni l'occasion aux personnes ayant une incapacité de se prendre en main et de faire leurs premiers pas vers leur réhabilitation. 
Joanne Beauchamps va régulièrement au Centre pour faire de la cuisine, de la couture, des exercices et même organiser des danses dans des salles publiques, à l'extérieur du Centre lui-même.

Cette initiative en dit long sur la volonté d'intégration des personnes vivant avec une incapacité. Pour ceux et celles qui se déplacent en fauteuil roulant ou qui vivent avec un handicap, il n'est désormais plus question de se cacher. Pourtant, il faut dire que si ces gens peuvent organiser une danse dans un endroit public, c'est qu'enfin il existe des endroits équipés de rampes et d'ascenseurs pour les accommoder.

Au début, quand j'organisais des événements en public, j'étais intimidée, car j'avais été habituée à vivre sous la direction ou la supervision d'une autre personne. Si je suis active aujourd'hui et que je suis capable de prendre des décisions par moi-même, c'est grâce à l'intervention de mon enseignante à l'école secondaire. Sans vouloir nuire à mon développement, mes parents m'ont toujours protégé et décidé à ma place ce qui était bon pour moi. Je crois qu'ils agissaient inconsciemment, sans aucune mauvaise intention. Je crois qu'ils voulaient simplement me faciliter la vie. Mais moi, j'ai compris que j'étais pourvue d'un cerveau et d'une intelligence et que j'étais capable de prendre des décisions, de réfléchir et de vivre.

Si les activités du Centre ont permis à Joanne d'acquérir son autonomie, elles ont aussi été un catalyseur pour Louise Grégoire.

Le Centre de projet de participation communautaire a été ma porte de salut. J'ai réalisé que je souffrais d'une incapacité émotive et psychologique, car je refusais d'accepter ma condition physique. Mes limites physiques emprisonnaient mes pensées.

Aujourd'hui, Louise n'est plus la même personne qu'au moment où elle appris la nouvelle de sa maladie. Elle adore parler même si la maladie affecte ses glandes au niveau des cordes vocales. Elle se rend régulièrement au Centre pour y rencontrer ses amies. Louise 
fait aussi des exercices et participe à des groupes de rencontre pour se tenir au courant des récents traitements médicaux de sa maladie et profite de l'occasion pour échanger des idées et des expériences vécues.

Sans les projets de participation communautaire, je resterais enfermée à la maison comme j'y étais habituée et je passerais des heures à dépérir.

La levée du rideau!

Je veux aller magasiner... je veux aller au restaurant... je veux marcher un jour... je vis, donc j'existe!

Ces phrases reviennent comme des leitmotivs dans une vidéo produite l'année dernière dans le cadre du programme d'approche du Centre. La production de cette vidéo promotionnelle a changé la vie de Louise Grégoire et de Joanne Beauchamps, car elle lève le voile sur la vie des personnes ayant une incapacité. Selon les participants et participantes à ce projet, il n'était plus question de demeurer dans l'ombre. Au contraire, le temps était venu de faire la lumière sur leurs conditions et de montrer à la société qu'ils sont normaux. Eh oui! La vidéo d'environ quinze minutes laisse parler ceux et celles qui ont choisi de se prendre en main en demandant de l'aide à ceux qui travaillent au Centre. Selon la directrice du centre, Margaret Borley, ce genre d'initiatives est nécessaire pour briser les préjugés, informer les gens des services disponibles et renseigner la population sur la réalité des personnes vivant avec une incapacité.

C'est le seul document vidéo duquel personne n'a été exclue. Toutes les personnes ayant une incapacité et qui ont bien voulu participer à sa production ont été admises.

Normalement, on mettait de coté les gens avec un problème d'élocution et de prononciation, car il était difficile de les entendre et de les comprendre. Or, sur la vidéo du Centre, tout le monde, sans exception, a été sollicité. Aux dires de Margaret Borley:

C'est déjà une preuve qu'il faut briser les préjugés à l'endroit des personnes ayant une incapacité et passer 
par-dessus les limites dans lesquelles la société les a toujours placées. Il faut montrer aux gens la réalité et la vie de ceux et celles qui vivent avec une incapacité. C'est déjà un pas dans la bonne direction, celle de l'intégration sociale de tous, et ce, sans exceptions.

\section{Étape 1: Briser 1'ignorance}

Selon Louise Grégoire et Joanne Beauchamps, deux des vedettes de la vidéo, il y encore beaucoup d'ignorance au sein de la population. Selon Louise, les gens ont peur. Certains vont même jusqu'à se demander pourquoi les personnes vivant avec une incapacité décident tout à coup de sortir de leur isolement et de se montrer au grand jour.

Certaines personnes semblent même craintives et se questionnent sur ce que nous voulons. Ce que nous voulons, c'est de vivre et montrer au monde que nous existons. C'est tout! C'est pourtant simple. Si je veux aller magasiner,je devrais être en mesure d'y aller comme tout le monde, car je peux me déplacer et parler comme tout le monde.Toutefois,j'éprouve encore de la difficulté à accepter que les gens puissent avoir peur ou se sentir mal à l'aise d'ouvrir une porte lors de mon passage. C'est peut-être qu'ils ont peur de s'ouvrir eux-mêmes à notre réalité et de voir qu'il est possible de partager nos existences en tant que membres de la même société.

Louise Grégoire estime que sa participation à la vidéo promotionnelle du Centre lui a ouvert les yeux. Autrefois fragile et inquiète, elle comprend maintenant l'attitude de ceux et celles qui ont de la difficulté à s'ouvrir les yeux sur les incapacités, car elle a vécu elle-même ce phénomène. Pourtant, aujourd'hui, Louise se dit prête et déterminée à s'intégrer à la société. Tout ce qu'il reste à faire, c'est de s'assurer que la société sera également prête à les accepter.

Les gens ignorent trop souvent encore l'incapacité et les conditions limitées auxquelles nous sommes confrontés à tous les 
jours. Le comprendre, c'est une question de respect entre deux individus, voilà ce que nous exigeons.

La meilleure façon de briser l'ignorance, c'est d'abord d'informer les gens et de les éduquer au sujet de la réalité des personnes qui vivent avec une incapacité physique ou mentale. La vidéo a d'ailleurs été diffusée à plusieurs reprises pour montrer à la population que ceux et celles ayant une incapacité ne sont pas des personnages étranges. Dans le fond, ils recherchent la même chose que tout le monde: vivre dans le respect et grandir tout en sachant qu'ils ont un rôle à jouer dans la société.

Le seul problème avec la vidéo se situe au niveau des médias. Les grands réseaux de télévision ne l'ont toujours pas diffusée. Leurs programmations trop rigides rendent la tâche difficile. Pour l'instant, il est donc impossible de diffuser à grande échelle la vidéo produite par le Centre. Mais les efforts ne s'arrêtent pas là, puisque la directrice Margaret Borley a déjà prévu un programme de sensibilisation pour les écoles de niveau primaire dans la région de Sudbury. La vidéo sera présentée dans les salles de classe en compagnie d'une des personnes ayant participé à sa production. C'est ce moment que Louise Grégoire a choisi afin d'agir.

Je veux dire au monde et aux jeunes en particulier, d'éviter d'avoir peur et de poser les questions auxquelles ils ont toujours voulu avoir une réponse. J'aimerais leur parler de ce que j'ai vécu, comment j'ai réussi à surmonter ma peur au sujet de ma condition physique. De cette façon, je crois qu'il nous sera possible de vivre comme une seule société: celle où l'on trouve le respect entre les personnes dites normales et celles qui vivent avec des limites physiques et psychologiques. C'est important de dire également que chacun a un rôle à jouer au sein de la société et ça, c'est seulement par l'éducation que nous pourrons franchir cette barrière. 


\section{Étape 2: Le monde du travail}

La société actuelle accorde beaucoup d'importance à la productivité des individus. Cette emphase s'érige comme un mur et rappelle qu'il reste encore plusieurs étapes à franchir avant de parvenir à l'intégration complète des personnes ayant une incapacité.Tous les secteurs d'activités, que ce soit le niveau médical ou encore certains services publics, ont réussi, au cours des dernières années, à s'adapter à la réalité de ceux et celles ayant une limitation physique. En général, la société accepte de plus en plus la condition des personnes vivant avec une incapacité, mais là où le problème persiste, c'est au niveau du marché du travail.

À l'heure actuelle, la conception dominante des entreprises affirme qu'un individu limité physiquement est moins productif qu'un autre travailleur. On refuse donc de l'embaucher, à moins que son emploi soit directement subventionné par les programmes spéciaux mis sur pied par l'État. Pourtant, il y a des personnes vivant avec une incapacité qui sont productives, sauf qu'on peut les compter sur les doigts d'une main! La plupart de ceux qui occupent un emploi, malgré leurs limites physiques ou psychologiques, sont des gens qui ont déjà acquis une certaine expérience de travail dans le passé. De plus, il s'agit surtout de personnes qui n'éprouvent aucune difficulté au niveau de l'élocution verbale. Il $\mathrm{y}$ a donc un travail énorme pour changer la perception des dirigeants d'entreprises afin de permettre aux personnes limitées physiquement de prendre leur place au sein de la société. Il faut accepter les gens comme ils sont. Il faut les traiter sur une base individuelle, car chaque personne est différente. Comme société, il faut cesser de mettre toutes les personnes vivant avec une incapacité dans le même bateau.

Ce que nous tentons de faire dans la vidéo promotionnelle, c'est d'illustrer à quel point chaque individu doit être traité avec dignité et d'être en mesure de reconnaitre ses valeurs et son potentiel. C'est aussi de cette façon que nous essayons de vivre. Il $\mathrm{y}$ a bien sûr d'énormes problèmes, tant au niveau technique 
qu'au niveau des habiletés de chaque individu, sauf que l'harmonie sociale passe encore par le respect des êtres humains.

\section{Étape $3:$ «J'existe!»}

Le Centre donne la possibilité aux personnes vivant avec une incapacité de regagner la confiance nécessaire pour leur permettre de s'intégrer à la société. C'est la confiance personnelle qui est l'arme la plus utile pour affronter les préjugés et les pressions sociales, mais aussi pour se prouver à soi-même que chacun a sa place au sein d'une communauté. Comme le dit Margaret Borley:

Nous pouvons être vos jambes et vos bras, mais il reste que c'est à chaque individu d'organiser sa vie pour être capable de vivre normalement selon ses capacités.

La perception du public a changé, l'embâcle a cédé et il n'est plus rare de voir quelqu'un se déplacer en fauteuil roulant sur les trottoirs ou dans tout autre endroit public.

En 1982, je me souviens que j'étais devant un centre d'achat de Sudbury et que mon amie Mary attendait patiemment l'autobus. Elle était assise dans son fauteuil roulant, lorsqu'un individu s'est approché d'elle pour déposer un billet de deux dollars sur ses genoux. Mary était tellement surprise de ce geste qu'elle a éclaté de rire!

Auparavant, le médecin posait toujours des questions au sujet d'une personne assise dans un fauteuil roulant, à celle qui l'accompagnait, comme si celle qui vivait avec une incapacité physique ne pouvait parler ou réfléchir par elle-même. C'est étonnant, mais il faut simplement constater à quel point, en peu de temps, la situation a beaucoup changé. Les gens ne font plus ça aujourd'hui. C'est la preuve que ce n'est plus un phénomène nouveau. La société a compris que ceux et celles qui vivent avec une incapacité ne sont pas si différents finalement. 
Il est important de mentionner que les personnes vivant avec une incapacité représente une infime partie de la population. Et comme leur nombre est faible, les compagnies ne sont pas intéressées à investir des sommes importantes pour améliorer les prothèses ou les autres produits utilisés par les personnes qui vivent avec des limites physiques.

Dans la vidéo promotionnelle, le mot exister revient à plusieurs occasions. Pour les personnes vivant avec une incapacité, ce terme vient confirmer l'importance d'obtenir une place dans la société au même titre que tout le monde. Ce besoin de s'intégrer à la société s'est fait sentir chez Louise Grégoire et Joanne Beauchamps. Pour atteindre leurs objectifs, elles ont pris les moyens qui étaient à leur disposition pour s'intégrer à la société. Aujourd'hui, elles vivent pleinement leur vie.

Il reste encore beaucoup d'étapes à franchir et d'épreuves à surmonter. Toutefois, l'intégration sociale des personnes vivant avec une incapacité c'est d'abord le défi à relever de toute une société. Avec le soutien des gens du Centre de participation communautaire et les outils techniques pour faciliter la mobilité ou l'élocution des gens, Louise et Joanne ont trouvé la force pour vivre pleinement leur vie. Et c'est l'expression d'une volonté sincère de l'être humain dans sa recherche d'une société où tous seront égaux. 